\title{
Weak difference property of functions with the Baire property
}

by

\author{
Tamás Mátrai (Budapest)
}

\begin{abstract}
We prove that the class of functions with the Baire property has the weak difference property in category sense. That is, every function for which $f(x+h)-f(x)$ has the Baire property for every $h \in \mathbb{R}$ can be written in the form $f=g+H+\phi$ where $g$ has the Baire property, $H$ is additive, and for every $h \in \mathbb{R}$ we have $\phi(x+h)-\phi(x) \neq 0$ only on a meager set. We also discuss the weak difference property of some subclasses of the class of functions with the Baire property, and the consistency of the difference property of the class of functions with the Baire property.
\end{abstract}

1. Introduction. Let $\mathbb{R}$ denote the set of real numbers and let $F$ be a class of real-valued functions on $\mathbb{R}$. We say that $F$ has the difference property if every function $f: \mathbb{R} \rightarrow \mathbb{R}$ for which

$$
f(x+h)-f(x) \in F \quad \text { for every } h \in \mathbb{R}
$$

can be written in the form

$$
f=g+H
$$

where $g \in F$ and $H$ is additive, that is, $H(x+y)=H(x)+H(y)$ for every $x, y \in \mathbb{R}$. For real $h$ we shall write

$$
\Delta_{h} f(x)=f(x+h)-f(x)
$$

for the difference functions of $f$.

Many function classes have the difference property, but the class of Lebesgue measurable functions does not if we assume the continuum hypothesis (see [5] or [6] for details). However, it was conjectured by Erdős that every function $f: \mathbb{R} \rightarrow \mathbb{R}$ for which $\Delta_{h} f$ is measurable for every $h \in \mathbb{R}$ is of the form $f=g+H+\phi$ where $g$ is measurable, $H$ is additive and for every $h \in \mathbb{R}, \phi(x+h)-\phi(x)=0$ almost everywhere (with respect to Lebesgue measure). This led to the definition of the weak difference property.

2000 Mathematics Subject Classification: Primary 26A21. 
We say that a class $F$ has the weak difference property if every function $f$ for which

$$
\Delta_{h} f(x) \in F \quad \text { for every } h \in \mathbb{R}
$$

can be written in the form

$$
f=g+H+\phi
$$

where $g \in F, H$ is additive and for every $h \in \mathbb{R}, \Delta_{h} \phi=0$ almost everywhere.

The conjecture of Erdös, namely the weak difference property of the class of Lebesgue measurable functions, and many of its consequences, were proved by M. Laczkovich [5].

The weak difference property in category sense was introduced in [1]. If a property $P(x)$ holds for every $x \in \mathbb{R}$ except a meager set of $x$ 's then we say that $P(x)$ holds $\mathcal{M}$-almost everywhere (for short $\mathcal{M}$-a.e.) or for $\mathcal{M}$-almost every $x$, where $\mathcal{M}$ stands for the class of meager subsets of $\mathbb{R}$. Analogously we say that a class $F$ has the weak difference property in category sense if every function $f$ for which

$$
\Delta_{h} f(x) \in F \quad \text { for every } h \in \mathbb{R}
$$

can be written in the form

$$
f=g+H+\phi
$$

where $g \in F, H$ is additive and for every $h \in \mathbb{R}, \Delta_{h} \phi=0 \mathcal{M}$-almost everywhere. The functions $\phi$ of this kind will be called null.

In [1] some problems were formulated on the analogy of the classical weak difference property problems. One of these, the counterpart of the result of M. Laczkovich for Lebesgue measurable functions, that is, the weak difference property in category sense of the class of functions with the Baire property, is proved in this paper. (A real-valued function has the Baire property if for every $b \in \mathbb{R}$ the set $\{x \in \mathbb{R}: f(x)<b\}$ has the Baire property, that is, it can be obtained as the symmetric difference of an open and a meager set.) Once this is done, it will be a simple task to establish the weak difference property of some subclasses of functions having the Baire property. We will prove two theorems:

THEOREM 1.1. Let $f: \mathbb{R} \rightarrow \mathbb{R}$ be a function with uniformly essentially bounded difference functions, that is, for some $K \in \mathbb{R}$,

$$
\left|\Delta_{h} f(x)\right|<K \quad \mathcal{M} \text {-a.e. }
$$

for every $h \in \mathbb{R}$. If $\Delta_{h} f$ has the Baire property for every $h \in \mathbb{R}$ then

$$
f=g+\phi
$$

where $g$ has the Baire property and $\phi$ is null.

This answers Problem 2.2 of [1]. 
THEOREM 1.2. Let $f: \mathbb{R} \rightarrow \mathbb{R}$ be an arbitrary function. If $\Delta_{h} f$ has the Baire property for every $h \in \mathbb{R}$ then

$$
f=g+H+\phi
$$

where $g$ has the Baire property, $H$ is additive and $\phi$ is null. That is, the class of functions with the Baire property has the weak difference property in category sense.

In [1] it was observed that similarly to the proof in [3] of the weak difference property for the class of functions a.e. equal to a continuous function (with respect to Lebesgue measure), the weak difference property in category sense of the class of functions with the Baire property would imply this property for the class of functions that equal a continuous function $\mathcal{M}$-almost everywhere. Therefore Theorem 1.2 has the following corollary.

Corollary 1.3 (Problem 2.1 in [1]). The class of functions $\mathcal{M}$-a.e. equal to a continuous function has the weak difference property in category sense.

It was observed in [6] that the consistency of the difference property of the class of functions with the Baire property is also a corollary of Theorem 1.2.

Corollary 1.4 (Problem 8.4 in [6]). It is consistent with ZFC that the class of functions with the Baire property has the difference property.

Independently of our results, this statement was recently proved in [2].

2. Preliminaries. In the proofs we will need two well known theorems (see e.g. [4] or [7]).

ThEOREM 2.1 (Kuratowski-Ulam). Let $k+l=n$ and let $H \subset \mathbb{R}^{n}$ be meager. Then there is a meager set $H_{0} \subset \mathbb{R}^{k}$ such that $\left(\{x\} \times \mathbb{R}^{l}\right) \cap H$ is meager for every $x \in \mathbb{R}^{k} \backslash H_{0}$. Conversely, if $H$ is of second category and has the Baire property then there is a set $H_{0} \subset \mathbb{R}^{k}$ of second category such that $\left(\{x\} \times \mathbb{R}^{l}\right) \cap H$ is of second category for every $x \in H_{0}$.

Definition 2.2. Let $H$ be a subset of $\mathbb{R}$ and let $I \subset \mathbb{R}$ be an open interval. We say that $H$ is of second category everywhere in $I$ if for every nonempty open interval $J \subset I$ the set $H \cap J$ is of second category.

THEOREM 2.3 (Banach). If $H \subset \mathbb{R}$ is of second category then it is of second category everywhere in a suitable nonempty open interval of $\mathbb{R}$.

We also use a classical result on the difference property. Its proof can be found e.g. in [6].

Theorem 2.4 (Stability Theorem of Hyers). Let $f: \mathbb{R} \rightarrow \mathbb{R}$ be such that

$$
|f(x+y)-f(x)-f(y)| \leq K
$$


for a nonnegative constant $K$. Then there is an additive function $H$ such that

$$
|f-H| \leq K \text {. }
$$

In the proofs $\mathbb{Q}, \mathbb{N}$ and $\mathbb{Z}$ will stand for the sets of rationals, positive integers and integers respectively, and $\lambda$ will denote the Lebesgue measure. For $A, B \subset \mathbb{R}, h \in \mathbb{R}$,

$$
A \triangle B=(A \backslash B) \cup(B \backslash A)
$$

will denote the symmetric difference of $A$ and $B$, while

$$
A+h=\{x+h: x \in A\}, \quad A+A=\{x+y: x, y \in A\} .
$$

In order to avoid the use of multiple parentheses we adopt the convention that

$$
A \cap B+h=(A \cap B)+h .
$$

For $x \in \mathbb{R}^{n}$ and $r>0$ the open ball centered at $x$ with radius $r$ will be denoted by $B(x, r)$. For a function $f: \mathbb{R} \rightarrow \mathbb{R}$ let

$$
[f<b]=\{x \in \mathbb{R}: f(x)<b\} .
$$

The sets $[f>b],[f \leq b]$ and $[f \geq b]$ are defined analogously.

We will also use the following notation. For $K: \mathbb{R} \times \mathbb{R} \rightarrow \mathbb{R}$ and $y \in \mathbb{R}$,

$$
K^{y}(x)=K(x, y)
$$

denotes the horizontal section of $K$ on $\mathbb{R} \times\{y\}$. The same is defined for sets, that is, for $S \subset \mathbb{R} \times \mathbb{R}$ we write

$$
S^{y}=S \cap(\mathbb{R} \times\{y\}) .
$$

3. Bounded functions. In the proof of Theorem 1.1 we will follow some ideas of M. Laczkovich [5]. We will need a kind of "norm" in order to measure the proximity of two functions having the Baire property.

Definition 3.1. For a set $H \subset \mathbb{R}$ with the Baire property let

$$
\begin{aligned}
\mathcal{N}(H)=\bigcup\{I \subset \mathbb{R}: I \text { is an open interval } \\
\text { and } H \text { is of second category everywhere in } I\} .
\end{aligned}
$$

It is easy to check that $\mathcal{N}(H)$ is an open set. The Banach Theorem and the Baire property of $H$ imply that $H \triangle \mathcal{N}(H)$ is meager.

Definition 3.2. Let $f: \mathbb{R} \rightarrow \mathbb{R}$ be a periodic function with period 1 having the Baire property. Let $f^{+}=\max \{f, 0\}$ and $f^{-}=\max \{-f, 0\}$ be the positive and negative parts of $f$. Our "norm" will be

$$
\|f\|=\int_{0}^{1} \lambda\left(\mathcal{N}\left(\left[f^{+}>t\right]\right) \cap[0,1]\right) d t+\int_{0}^{1} \lambda\left(\mathcal{N}\left(\left[f^{-}>t\right]\right) \cap[0,1]\right) d t .
$$


The integrals exist since the integrands are nonincreasing. It is easy to see that the triangle inequality does not hold for $\|\cdot\|$. This is the major source of technical difficulties.

Lemma 3.3. Let $\varepsilon>0$, let $G_{1}, G_{2}, \ldots$ be a sequence of measurable subsets of $[0,1]$ such that

$$
\lambda\left(G_{i}\right)>\varepsilon \quad \text { for } i=1,2, \ldots,
$$

and let $\left(h_{i}\right)$ be a sequence of reals converging to zero. Define $T: \mathbb{R} \rightarrow$ $\mathbb{N} \cup\{0\} \cup\{\infty\}$ by

$$
\begin{aligned}
T(x):=\sup \{k & \in \mathbb{N} \cup\{0\}: \exists\left(j_{1}, \ldots, j_{k}\right) \in \mathbb{N}^{k}: \\
x & \in \underbrace{\left(\left(\ldots\left(\left(G_{j_{1}}+h_{j_{1}}\right) \cap G_{j_{2}}+h_{j_{2}}\right) \cap \ldots\right) \cap G_{j_{k}}+h_{j_{k}}\right\} .}_{k-1}
\end{aligned}
$$

Then

$$
\lambda([T=\infty]) \geq \varepsilon .
$$

Proof. The function $T$ is clearly measurable. First we prove by induction that $T \leq K$ is impossible for any $K \in \mathbb{N}$.

The assumption $T \leq 1$ would imply

$$
\bigcup_{i=1}^{\infty} G_{i} \cap \bigcup_{i=1}^{\infty}\left(G_{i}+h_{i}\right)=\emptyset \text {. }
$$

On the other hand, since $\left(h_{i}\right)$ converges to zero, for $I$ sufficiently large we have

$$
\lambda\left(\left(\bigcup_{i=1}^{\infty} G_{i}\right) \triangle\left(\left(\bigcup_{i=1}^{\infty} G_{i}\right)+h_{I}\right)\right)<\varepsilon / 2,
$$

thus using the fact that $\lambda\left(G_{I}+h_{I}\right)>\varepsilon$ we get

$$
\lambda\left(\left(G_{I}+h_{I}\right) \cap \bigcup_{i=1}^{\infty} G_{i}\right)>\varepsilon / 2,
$$

contradicting (1).

Let now $K \geq 2$ and suppose that $T \leq K-1$ is impossible and that $T \leq K$. The assumption $T \leq K$ implies

$$
\bigcup_{i=1}^{\infty} G_{i} \subset[T \leq K-1] .
$$

Again, for $I$ large enough and any $j>I$ we have

$$
\lambda\left([T \leq K-1] \triangle\left([T \leq K-1]+h_{j}\right)\right)<\varepsilon / 2,
$$

SO

$$
\lambda\left(\left(G_{j}+h_{j}\right) \cap[T \leq K-1]\right)>\varepsilon / 2 .
$$


For $i=1,2, \ldots$ let

$$
\widetilde{G}_{i}=\left(G_{I+i}+h_{I+i}\right) \cap[T \leq K-1]-h_{I+i}, \quad \widetilde{h}_{i}=h_{I+i} .
$$

Then $\widetilde{G}_{i} \subset G_{I+i}, \widetilde{G}_{i}$ is measurable and $\lambda\left(\widetilde{G}_{i}\right)>\varepsilon / 2$ by (2). Now one can define the function $\widetilde{T}$ for this sequence of sets and reals the same way $T$ was defined. Since $\widetilde{G}_{i} \subset G_{I+i}$ and $\widetilde{h}_{i}=h_{I+i}$ it is easy to see that $\widetilde{T} \leq T$. From (3) we get

$$
[\widetilde{T} \neq 0] \subset[T \leq K-1] .
$$

Hence $\widetilde{T} \leq K-1$, which contradicts the induction assumption. Thus we have proved that $T$ cannot be bounded.

Suppose now that for some $\delta>0$ we have

$$
\lambda([T=\infty])<\varepsilon-\delta .
$$

Let $K$ be such that $\lambda([T \geq K])<\varepsilon-\delta / 2$, and so

$$
\lambda\left(G_{i} \cap[T<K]\right)>\delta / 2 .
$$

Again, if $I$ is sufficiently large then for any $j>I$ we have

$$
\lambda\left([T<K] \triangle\left([T<K]+h_{j}\right)\right)<\delta / 4,
$$

so

$$
\lambda\left(\left(G_{j} \cap[T<K]+h_{j}\right) \cap[T<K]\right)>\delta / 4 .
$$

We continue as above. Let

$$
\widetilde{G}_{i}=\left(G_{I+i} \cap[T<K]+h_{I+i}\right) \cap[T<K]-h_{I+i}, \quad \widetilde{h}_{i}=h_{I+i} .
$$

Since by (4) we have $\lambda\left(\widetilde{G}_{i}\right)>\delta / 4$, and again $\widetilde{G}_{i} \subset G_{I+i}$ is measurable, we can define the function $\widetilde{T}$ for this sequence of open sets and reals as before, and we get $\widetilde{T}<K$. This contradicts the impossibility of boundedness and proves the statement.

LemMA 3.4. Let $\varepsilon>0$, let $G_{1}, G_{2}, \ldots$ be a sequence of open subsets of $[0,1]$ such that

$$
\lambda\left(G_{i}\right)>\varepsilon \quad \text { for } i=1,2, \ldots,
$$

and let $\left(h_{i}\right)$ be a sequence of reals converging to zero. Then for any $N \in \mathbb{N}$ one can find an open interval $I_{N}$ and a sequence $\left(j_{1}, \ldots, j_{N+1}\right) \in \mathbb{N}^{N+1}$ such that

$$
I_{N} \subset \underbrace{((\ldots)}_{N}\left(\left(G_{j_{1}}+h_{j_{1}}\right) \cap G_{j_{2}}+h_{j_{2}}\right) \cap \ldots) \cap G_{j_{N+1}}+h_{j_{N+1}} .
$$

Proof. Using the notations and statement of Lemma 3.3 we find that $[T>N]$ is nonempty. Since

$$
\begin{array}{r}
{[T>N]=\bigcup\{\underbrace{\left(\left(\ldots\left(\left(G_{j_{1}}+h_{j_{1}}\right) \cap G_{j_{2}}+h_{j_{2}}\right) \cap \ldots\right) \cap G_{j_{N+1}}+h_{j_{N+1}}:\right.}_{N}} \\
\left.\left(j_{1}, \ldots, j_{N+1}\right) \in \mathbb{N}^{N+1}\right\},
\end{array}
$$


we deduce that for some $\left(j_{1}, \ldots, j_{N+1}\right)$ the open set

$$
\underbrace{((\ldots)}_{N}\left(\left(G_{j_{1}}+h_{j_{1}}\right) \cap G_{j_{2}}+h_{j_{2}}\right) \cap \ldots) \cap G_{j_{N+1}}+h_{j_{N+1}}
$$

is also nonempty.

LEMMA 3.5. Let $f: \mathbb{R} \rightarrow \mathbb{R}$ be periodic with period 1 and suppose that $\Delta_{h} f$ is uniformly essentially bounded and has the Baire property for every $h$. Then

$$
\left\|\Delta_{h_{n}} f\right\| \rightarrow 0 \quad \text { if } h_{n} \rightarrow 0
$$

Proof. Let $\left|\Delta_{h} f\right|<K \mathcal{M}$-a.e. Suppose that there is an $\varepsilon>0$ and a sequence $\left(h_{n}\right)$ converging to zero such that

$$
\left\|\Delta_{h_{n}} f\right\|>\varepsilon \quad(\forall n \in \mathbb{N}) .
$$

Using the fact that for any nonincreasing function $g:[0,1] \rightarrow[0,1]$ we have

$$
\int_{0}^{1} g \leq a+g(a)
$$

for every $a \in[0,1]$, we find that for any $n \in \mathbb{N}$ either

$$
\lambda\left(\mathcal{N}\left(\left[\left(\Delta_{h_{n}} f\right)^{+}>\varepsilon / 4\right]\right)\right)>\varepsilon / 4 \text { or } \lambda\left(\mathcal{N}\left(\left[\left(\Delta_{h_{n}} f\right)^{-}>\varepsilon / 4\right]\right)\right)>\varepsilon / 4 .
$$

By choosing a subsequence we may suppose that the same case holds for every $n \in \mathbb{N}$. The two cases can be treated in the same way, so we consider only the first one.

So suppose that

$$
\lambda\left(\mathcal{N}\left(\left[\left(\Delta_{h_{n}} f\right)^{+}>\varepsilon / 4\right]\right)\right)>\varepsilon / 4 \quad \text { for every } n \in \mathbb{N} .
$$

Let

$$
G_{n}=\mathcal{N}\left(\left[\left(\Delta_{h_{n}} f\right)^{+}>\varepsilon / 4\right]\right)=\mathcal{N}\left(\left[\Delta_{h_{n}} f>\varepsilon / 4\right]\right) .
$$

These are open sets with $\lambda\left(G_{n}\right)>\varepsilon / 4$ so by applying Lemma 3.4 for $N=$ $[4 K / \varepsilon+1]$ (where [.] stands for the integer part) we get a nonempty open interval $I_{N}$ and a sequence $\left(j_{1}, \ldots, j_{N+1}\right)$ with the properties of Lemma 3.4. Let

$$
h=\sum_{i=1}^{N+1} h_{j_{i}}
$$

We claim that for $\mathcal{M}$-almost every $x \in I_{N}-h$,

$$
\Delta_{h} f \geq N \varepsilon / 4 \geq K
$$


which is a contradiction. We have

$$
\begin{aligned}
\Delta_{h} f(x)= & \Delta_{h_{j_{N+1}}} f\left(x+h_{j_{1}}+\ldots+h_{j_{N}}\right) \\
& +\Delta_{h_{j_{N}}} f\left(x+h_{j_{1}}+\ldots+h_{j_{N-1}}\right)+\ldots \\
& +\Delta_{h_{j_{k}}} f\left(x+h_{j_{1}}+\ldots+h_{j_{k-1}}\right)+\ldots \\
& +\Delta_{h_{j_{2}}} f\left(x+h_{j_{1}}\right)+\Delta_{h_{j_{1}}} f(x) .
\end{aligned}
$$

For every $x \in I_{N}-h$ we have $x+h \in I_{N}$, thus (5) yields

$$
x+h-h_{j_{N+1}}-\ldots-h_{j_{k}} \in G_{j_{k}} .
$$

Since

$$
x+h-h_{j_{N+1}}-\ldots-h_{j_{k}}=x+h_{j_{1}}+\ldots+h_{j_{k-1}},
$$

by the definition of $G_{j_{k}}$ we obtain

$$
\Delta_{h_{j_{k}}} f\left(x+h_{j_{1}}+\ldots+h_{j_{k-1}}\right)>\varepsilon / 4 \quad \mathcal{M} \text {-a.e. on } I_{N}-h
$$

for every $1 \leq k \leq N+1$, which implies (6).

The following lemma is a straightforward consequence of the Baire category theorem.

Lemma 3.6. Let $S, T \subset[0,1] \times[0,1]$ be such that $T^{y}$ and $S^{y}$ are open for every $y \in[0,1]$. If $(T \backslash S)^{y}$ is of second category for every $y \in Y$ where $Y$ is nonmeager then there are $u, v \in \mathbb{Q}$ and $Y^{\prime} \subset Y$ of second category such that

$$
(u, v) \subset(T \backslash S)^{y} \quad \text { for every } y \in Y^{\prime} .
$$

Lemma 3.7. Let $K:[0,1]^{2} \rightarrow \mathbb{R}$ have the following properties:

(i) $K^{y}$ has the Baire property for every $y \in[0,1]$;

(ii) $\left\|K^{y_{n}}-K^{y}\right\| \rightarrow 0$ if $y_{n} \rightarrow y$ in $[0,1]$.

Then there is a lower semicontinuous function $G:[0,1]^{2} \rightarrow \mathbb{R}$ such that

$$
K^{y}-G^{y}=0 \quad \mathcal{M} \text {-a.e. }
$$

for $\mathcal{M}$-almost every $y \in[0,1]$.

Proof. For $q \in \mathbb{Q}$ let

$$
\begin{gathered}
N(q)=\bigcup_{y \in[0,1]} \mathcal{N}\left(\left[K^{y}>q\right]\right) \times\{y\}, \\
M(q)=\bigcup_{\left.(B(x, r) \backslash N(q))^{y} \text { is meager for } \mathcal{M} \text {-almost every } y \in[0,1]\right\} .}
\end{gathered}
$$

Let

$$
G(x, y)=\sup \{q \in \mathbb{Q}:(x, y) \in M(q)\} .
$$

As a supremum of lower semicontinuous functions $(M(q)$ is open), $G$ is lower semicontinuous. In order to prove the statement of the lemma it is enough 
to show that

$$
K^{y}-G^{y}=0 \quad \mathcal{M} \text {-a.e. }
$$

for $\mathcal{M}$-almost every $y \in[0,1]$.

Consider the following relation for subsets of $[0,1] \times[0,1]$. For $T, S \subset$ $[0,1] \times[0,1]$ we write $T \sim S$ if $(T \triangle S)^{y}$ is meager for $\mathcal{M}$-almost every $y \in[0,1]$. This is clearly an equivalence relation. We shall prove that

$$
[G>q] \sim[K>q]
$$

for every $q \in \mathbb{Q}$. This will complete the proof, since $K^{y}-G^{y} \neq 0$ on a set of second category for a nonmeager set of $y$ 's would imply that

$$
([G>q] \triangle[K>q])^{y}
$$

is of second category for a nonmeager set of $y$ 's with an appropriate $q \in \mathbb{Q}$.

We will prove the following chain of equivalences:

$$
[G>q] \sim M(q) \sim N(q) \sim[K>q] .
$$

We start with the middle one, that is, $M(q) \sim N(q)$.

It follows easily from the definition of $M(q)$ that $(M(q) \backslash N(q))^{y}$ is meager for $\mathcal{M}$-almost every $y \in[0,1]$. So we have to show that $(N(q) \backslash M(q))^{y}$ is meager for $\mathcal{M}$-almost every $y \in[0,1]$.

Suppose that $(N(q) \backslash M(q))^{y}$ is not meager for a set $Z_{1} \subset[0,1]$ of $y$ 's of second category. From the definition of $N(q)$ it is straightforward to see that for $\varepsilon$ sufficiently small even $(N(q+\varepsilon) \backslash M(q))^{y}$ is not meager for a set $Z_{2} \subset Z_{1}$ of $y$ 's of second category. Since the horizontal sections of both $M(q)$ and $N(q+\varepsilon)$ are open, by Lemma 3.6 we find that there exists an open interval $(u, v) \subset[0,1]$ with $u, v \in \mathbb{Q}$ and a set $Z_{3} \subset Z_{2}$ of second category such that

$$
(u, v) \subset(N(q+\varepsilon) \backslash M(q))^{y} \quad \forall y \in Z_{3} .
$$

By the Banach Theorem one can find an open interval $U$ such that $Z_{3}$ is of second category everywhere in $U$. Let $U^{\prime}$ be the middle third of $U$ and $Z_{4}=Z_{3} \cap U^{\prime}$. Choose $\varrho \in \mathbb{Q}$ such that

$$
0<\varrho<\frac{1}{2} \min \left\{\frac{1}{2}|u-v|, \operatorname{diam}\left(U^{\prime}\right)\right\} .
$$

Fix $x_{0}=(u+v) / 2$ and take any $y_{0} \in Z_{4}$. Since $\left(x_{0}, y_{0}\right) \notin M(q)$, for any $y_{0}^{\prime} \in \mathbb{Q}$ with $\left|y_{0}-y_{0}^{\prime}\right|<\varrho$ we have

$$
B\left(\left(x_{0}, y_{0}^{\prime}\right), \varrho\right) \not \subset M(q) \text {. }
$$

This implies that

$$
\left(B\left(\left(x_{0}, y_{0}^{\prime}\right), \varrho\right) \backslash N(q)\right)^{y}
$$

is not meager for a set of $y$ 's of second category, so

$$
\left(B\left(\left(x_{0}, y_{0}\right), 2 \varrho\right) \backslash N(q)\right)^{y}
$$


is also not meager for a set $V_{1} \subset U$ of $y$ 's of second category, since

$$
\left(B\left(\left(x_{0}, y_{0}^{\prime}\right), \varrho\right) \backslash N(q)\right)^{y} \subset\left(B\left(\left(x_{0}, y_{0}\right), 2 \varrho\right) \backslash N(q)\right)^{y} .
$$

Again by Lemma 3.6, since the horizontal sections of $B\left(\left(x_{0}, y_{0}\right), 2 \varrho\right)$ and $N(q)$ are open, we infer that there is an open interval $\left(u^{\prime}, v^{\prime}\right) \subset(u, v)$ and a set $V_{2} \subset V_{1}$ of second category such that

$$
\left(u^{\prime}, v^{\prime}\right) \times V_{2} \subset B\left(\left(x_{0}, y_{0}\right), 2 \varrho\right) \backslash N(q) .
$$

Now we have a set $Z_{3}$ dense in $U$ and a set $V_{2}$ somewhere dense in $U$. So we can take a sequence $\left(y_{n}\right) \subset V_{2}$ with $y_{n} \rightarrow y$ for some $y \in Z_{3}$. From (7) we have $K^{y}>q+\varepsilon \mathcal{M}$-a.e. on $\left(u^{\prime}, v^{\prime}\right) \subset(u, v)$, and from (8) we get $K^{y_{n}} \leq q$ $\mathcal{M}$-a.e. on $\left(u^{\prime}, v^{\prime}\right)$. Hence

$$
\left[K^{y}-K^{y_{n}}<\varepsilon\right] \cap\left(u^{\prime}, v^{\prime}\right)
$$

is meager, so

$$
\lambda\left(\mathcal{N}\left(\left[K^{y}-K^{y_{n}}\right] \geq \delta\right)\right) \geq \lambda\left(\left(u^{\prime}, v^{\prime}\right)\right) \quad \text { for every } 0<\delta<\varepsilon,
$$

thus

$$
\left\|K^{y}-K^{y_{n}}\right\| \geq \varepsilon \lambda\left(\left(u^{\prime}, v^{\prime}\right)\right) \quad \text { for every } n \in \mathbb{N} .
$$

This is a contradiction, hence $(N(q) \backslash M(q))^{y}$ is meager for $\mathcal{M}$-almost every $y \in[0,1]$ and so $M(q) \sim N(q)$.

The equivalence $N(q) \sim[K>q]$ follows easily from the definitions.

Finally we prove $[G>q] \sim M(q)$. For any $q \in \mathbb{Q}$ we have

$$
[G>q]=\left\{(x, y) \in[0,1]^{2}: \exists r>q:(x, y) \in M(r)\right\}=\bigcup_{r>q} M(r) .
$$

Since $M(r) \sim N(r)$ for every $r \in \mathbb{Q}$, we get

$$
\bigcup_{r>q} M(r) \sim \bigcup_{r>q} N(r)
$$

Since $N(q) \sim[K>q]$ for every $q \in \mathbb{Q}$ and $[K>q]=\bigcup_{r>q}[K>r]$, we have

$$
N(q) \sim[K>q] \sim \bigcup_{r>q}[K>r] \sim \bigcup_{r>q} N(r) .
$$

So we get

$$
M(q) \sim N(q) \sim \bigcup_{r>q} N(r) \sim \bigcup_{r>q} M(r) \sim[G>q],
$$

as stated.

Proof of Theorem 1.1. We can suppose that $f$ is periodic with period 1 . Indeed, let $f_{1}(x)=f(\{x\})$ where $\{x\}$ stands for the fractional part of $x$. Then $f-f_{1}$ is essentially bounded and has the Baire property since the 
difference functions of $f$ are uniformly essentially bounded and for $n \leq x<$ $n+1$ we have

$$
f_{1}-f=f(x-n)-f(x)=\Delta_{-n} f .
$$

This implies that the difference functions of $f_{1}$ are also uniformly essentially bounded, while

$$
\begin{aligned}
\Delta_{h} f_{1}(x) & =f_{1}(x+h)-f_{1}(x) \\
& =\left[f_{1}(x+h)-f(x+h)\right]+[f(x+h)-f(x)]+\left[f(x)-f_{1}(x)\right]
\end{aligned}
$$

shows the Baire property for every $h$. If $f_{1}=g_{1}+\phi$ where $g_{1}$ has the Baire property and $\phi$ is null then $f=g+\phi$, where $g=\left(f-f_{1}\right)+g_{1}$ also has the Baire property.

Suppose now that $f$ is periodic with period 1 and let

$$
K(x, y)=f(x+y)-f(x) .
$$

The equality

$$
K^{y_{n}}(x)-K^{y}(x)=f\left(x+y_{n}\right)-f(x+y)=\Delta_{y_{n}-y} f(x+y)
$$

and Lemma 3.5 imply that $\left\|K^{y_{n}}-K^{y}\right\| \rightarrow 0$ if $y_{n} \rightarrow y$. Thus using the periodicity of $K$ and Lemma 3.7 we get a Borel function $G: \mathbb{R} \times \mathbb{R} \rightarrow \mathbb{R}$ such that $K^{y}-G^{y}=0 \mathcal{M}$-a.e. for $\mathcal{M}$-almost every $y \in \mathbb{R}$. Let

$$
S(x, y)=K(x, y)-G(x, y)=f(x+y)-f(x)-G(x, y) .
$$

Then $S(x, y)=0 \mathcal{M}$-a.e. in $x$ for $\mathcal{M}$-almost every $y \in \mathbb{R}$. By the definition of $S$ we have

$$
\begin{aligned}
S(x, y+z) & =f(x+y+z)-f(x)-G(x, y+z), \\
-S(x+y, z) & =-f(x+y+z)+f(x+y)+G(x+y, z), \\
-S(x, y) & =-f(x+y)+f(x)+G(x, y) .
\end{aligned}
$$

By adding and setting

$$
L(x, y, z)=S(x, y+z)-S(x+y, z)-S(x, y)
$$

we get

$$
L(x, y, z)=-G(x, y+z)+G(x+y, z)+G(x, y),
$$

so $L$, as a difference of Borel functions, has the Baire property. Hence the fact that for $\mathcal{M}$-almost every $z \in \mathbb{R}$ and for $\mathcal{M}$-almost every $y \in \mathbb{R}$ we have $L(x, y, z)=0 \mathcal{M}$-a.e. in $x$ implies by the converse part of the KuratowskiUlam Theorem that

$$
L(x, y, z)=0 \quad \mathcal{M} \text {-a.e. in } \mathbb{R}^{3} \text {. }
$$

The Kuratowski-Ulam Theorem tells us that there exists a point $x_{0}$ such that for $\mathcal{M}$-almost every $z \in \mathbb{R}$ we have

$$
L\left(x_{0}, y, z\right)=0 \quad \text { for } \mathcal{M} \text {-almost every } y \in \mathbb{R} .
$$


However, for $\mathcal{M}$-almost every $z \in \mathbb{R}$ also $S\left(x_{0}+y, z\right)=0 \mathcal{M}$-a.e. in $y$, so for $\mathcal{M}$-almost every $z \in \mathbb{R}$ we have

$$
\begin{aligned}
& L\left(x_{0}, y, z\right)+S\left(x_{0}+y, z\right) \\
& \quad=S\left(x_{0}, y+z\right)-S\left(x_{0}, y\right)=0 \quad \text { for } \mathcal{M} \text {-a.e. } y \in \mathbb{R} .
\end{aligned}
$$

Let $Z$ denote the residual set from which $z$ can be chosen in (9). For every $h \in \mathbb{R}$, since $Z \cap(h-Z) \neq \emptyset$, there are $z_{1}, z_{2} \in Z$ such that $h=z_{1}+z_{2}$. Therefore for every $h \in \mathbb{R}$ we have

$$
\begin{aligned}
& S\left(x_{0}, y+h\right)-S\left(x_{0}, y\right) \\
= & {\left[S\left(x_{0}, y+z_{1}+z_{2}\right)-S\left(x_{0}, y+z_{2}\right)\right]+\left[S\left(x_{0}, y+z_{2}\right)-S\left(x_{0}, y\right)\right]=0 }
\end{aligned}
$$

for $\mathcal{M}$-almost every $y \in \mathbb{R}$.

Now we can define $g$ and $\phi$. By the definition of $S$ we have

$$
S\left(x_{0}, y\right)=f\left(x_{0}+y\right)-f\left(x_{0}\right)-G\left(x_{0}, y\right),
$$

so

$$
f(y)=S\left(x_{0}, y-x_{0}\right)+f\left(x_{0}\right)+G\left(x_{0}, y-x_{0}\right) .
$$

Let

$$
g(y)=f\left(x_{0}\right)+G\left(x_{0}, y-x_{0}\right), \quad \phi(y)=S\left(x_{0}, y-x_{0}\right) .
$$

The function $g$, as a section of the Borel function $G$, obviously has the Baire property. The function $\phi$ is null, since by (10),

$$
\Delta_{h} \phi(y)=S\left(x_{0}, y+h-x_{0}\right)-S\left(x_{0}, y-x_{0}\right)=0 \quad \text { for } \mathcal{M} \text {-a.e. } y \in \mathbb{R} \text {. }
$$

4. Unbounded functions. Theorem 1.1 allows us to find a decomposition $f=g+\phi$ if it is guaranteed in some way that $f$ contains no additive function. In the following our goal is to find an additive function in a general $f$.

Definition 4.1. Let $g: \mathbb{R} \rightarrow \mathbb{R}$ have the Baire property. For an open interval $I \subset \mathbb{R}$ we say that the induced oscillation of $g$ is less than $D$ in $I$ if there exists an $a \in \mathbb{R}$ such that $I \subset \mathcal{N}([|g-a|<D])$. For $x \in \mathbb{R}$ we say that the induced oscillation of $g$ is less than $D$ at $x$ if there is an open interval $I$ with $x \in I$ such that the induced oscillation of $g$ is less than $D$ in $I$. In this case we say that the fact that the induced oscillation of $g$ at $x$ is less than $D$ is witnessed by $I$.

Set

$$
X_{D}(g)=\{x \in \mathbb{R}: \text { the induced oscillation of } g \text { at } x \text { is less than } D\} .
$$

LEMma 4.2. For every $g: \mathbb{R} \rightarrow \mathbb{R}$ with the Baire property and $D>0$ the set $\mathbb{R} \backslash X_{D}(g)$ is nowhere dense. 
Proof. The open set

$$
\bigcup_{a \in \mathbb{Q}} \mathcal{N}([|g-a|<D])
$$

is contained in $X_{D}(g)$, so it is enough to prove that it is dense. Let $I$ be an open interval. By the Baire category theorem, for some $a \in \mathbb{Q}$ the set $[|g-a|<D] \cap I$ is of second category, which - together with the Baire property of $g$-implies

$$
\mathcal{N}([|g-a|<D]) \cap I \neq \emptyset .
$$

Definition 4.3. Let $f: \mathbb{R} \rightarrow \mathbb{R}$ be such that $\Delta_{h} f$ has the Baire property for every $h \in \mathbb{R}$, let $D>0$, and consider the following relation on $\mathbb{R}$ :

$$
y \sim_{D} z \quad \text { if } y \in X_{D}\left(\Delta_{z-y} f\right) .
$$

For an open interval $I$ with $y \in I$ we say that $y \sim_{D} z$ is witnessed by $I$ if $y \in X_{D}\left(\Delta_{z-y} f\right)$ is witnessed by $I$.

That is, $y \sim_{D} z$ if the induced oscillation of $f(t+z)-f(t+y)$ is less than $D$ at $t=0$. This relation is symmetric and reflexive, but not transitive. It is easy to check that instead of transitivity we have the following property.

LEMmA 4.4. If $t \sim_{D} y$ and $t \sim_{D} z$, both witnessed by $B(t, \varepsilon)$, then $y \sim_{2 D} z$ witnessed by $B(y, \varepsilon)$.

We also have the following property.

LEMMA 4.5. If $y \sim_{D} t$ and $y \sim_{D} \tau$, both witnessed by $B(y, \varepsilon)$, and $|t-\tau|$ $<\delta<\varepsilon$, then $y \sim_{2 D} y+t-\tau$ witnessed by $B(y, \varepsilon-\delta)$.

Proof. By definition, $y \sim_{D} t$ and $y \sim_{D} \tau$ witnessed by $B(y, \varepsilon)$ means that the induced oscillation of $\Delta_{t-y} f(x)$ and $\Delta_{\tau-y} f(x)$ is less than $D$ in $B(y, \varepsilon)$. So $|t-\tau|<\delta$ implies that the induced oscillation of $\Delta_{t-y} f(x+\tau-t)$ in $B(y, \varepsilon-\delta)$ is also less than $D$. Since

$$
\Delta_{\tau-y} f(x)-\Delta_{t-y} f(\tau-t+x)=\Delta_{\tau-t} f(x)
$$

we conclude that the induced oscillation of $\Delta_{\tau-t} f(x)$ is less than $2 D$ in $B(y, \varepsilon-\delta)$, as required.

Definition 4.6. Let $f: \mathbb{R} \rightarrow \mathbb{R}$ be such that $\Delta_{h} f$ has the Baire property for every $h \in \mathbb{R}$, and let $D>0$. For $t \in \mathbb{R}$ let

$$
E_{f, D}(t)=\left\{y \in \mathbb{R}: t \sim_{D} y\right\} .
$$

Lemma 4.7. Let $\left(x_{i}\right) \subset \mathbb{R}$. If

$$
E=\bigcup_{i=1}^{\infty} E_{f, D}\left(x_{i}\right)
$$


is dense in an interval then

$$
F=\bigcup_{i=1}^{\infty} E_{f, 2 D}\left(x_{i}\right)
$$

is of second category everywhere in $\mathbb{R}$.

Proof. Suppose that $E$ is dense in an open interval $I$ but $F$ is meager in an open interval $J$. We can suppose $\lambda(I)=\lambda(J) / 2$. Let $\left(e_{j}\right) \subset E$ be also dense in $I$. Then one can take a translation by an $h \in \mathbb{R}$ such that

$$
\left(e_{j}\right)+h \subset J \backslash F,
$$

that is, $(E+h) \cap(J \backslash F)$ is dense in $J$. We claim that for every $t \in(E+h)$ $\cap(J \backslash F)-h$ the induced oscillation of $\Delta_{h} f$ is not less than $D$ at $t$. Indeed, since the induced oscillation of $\Delta_{h} f$ less than $D$ at $t$ would imply $t \sim_{D}(t+h)$, it follows that if $t \sim_{D} x_{i}$ (such an $x_{i}$ exists because $t \in E$ ) then by Lemma 4.4 we would have $x_{i} \sim_{2 D} t+h$ and $t+h \in F$, which is not true. Since $(E+h) \cap(J \backslash F)-h$ is dense in an interval, this contradicts Lemma 4.2.

Lemma 4.8. Let $g: \mathbb{R} \rightarrow \mathbb{R}$ have the Baire property. Suppose that for some $\delta>0$, a nonnegative constant $K$ and a dense subset $Z$ of $B(0,2 \delta)$, for every $z \in Z$ we have

$$
\left|\Delta_{z} g\right|<K \quad \mathcal{M} \text {-a.e. in } B\left(q_{0}, \delta\right)
$$

for some $q_{0} \in \mathbb{R}$. Then the induced oscillation of $g$ in $B\left(q_{0}, \delta\right)$ is less than $K$.

Proof. Suppose that the induced oscillation of $g$ in $B\left(q_{0}, \delta\right)$ is not less than $K$. Then there is an $a \in \mathbb{R}$ such that both $[g-a>3 K / 4]$ and $[g-a<$ $-3 K / 4]$ are of second category in $B\left(q_{0}, \delta\right)$. The Baire property of $g$ implies that

$$
\mathcal{N}([g-a>3 K / 4]) \cap B\left(q_{0}, \delta\right) \text { and } \mathcal{N}([g-a<-3 K / 4]) \cap B\left(q_{0}, \delta\right)
$$

are nonempty, so by the density of $Z$ in $B(0,2 \delta)$ there are $z \in Z$ and $x \in \mathcal{N}([g-a<-3 K / 4])$ such that $x+z \in \mathcal{N}([g-a>3 K / 4])$. From this and the Baire property of $g$ we have

$$
\left|\Delta_{z} g\right|>3 K / 2
$$

$\mathcal{M}$-a.e. in the nonempty open set

$$
(\mathcal{N}([g-a>3 K / 4])-z) \cap \mathcal{N}([g-a<-3 K / 4]) .
$$

This is a contradiction.

Proof of Theorem 1.2. Let $f: \mathbb{R} \rightarrow \mathbb{R}$ be such that $\Delta_{h} f$ has the Baire property for every $h \in \mathbb{R}$. By the reflexivity of $\sim_{D}$, the set

$$
\bigcup_{q \in \mathbb{Q}} E_{f, 1}(q)
$$


contains $\mathbb{Q}$, so it is dense. Then, by Lemma 4.7 for $\left(x_{i}\right)=\mathbb{Q}, D=1$, there is a $q_{0} \in \mathbb{Q}$ such that $E_{f, 2}\left(q_{0}\right)$ is of second category. Since

$$
E_{f, 2}\left(q_{0}\right)=\bigcup_{n \in \mathbb{N}}\left\{x \in \mathbb{R}: q_{0} \sim_{2} x \text { witnessed by } B\left(q_{0}, 1 / n\right)\right\},
$$

there exists an $n \in \mathbb{N}$ such that the set

$$
E^{n}=\left\{x \in \mathbb{R}: q_{0} \sim_{2} x \text { witnessed by } B\left(q_{0}, 1 / n\right)\right\}
$$

is of second category. By the Banach Theorem one can find an open interval $B\left(x_{0}, 24 \varrho\right)$ in which $E^{n}$ is of second category everywhere. We can suppose $24 \varrho<1 / n$. Let $E=E^{n} \cap B\left(x_{0}, 24 \varrho\right)$.

From Lemma 4.5 for every $t, \tau \in E$ with $|t-\tau|<8 \varrho$ we have $q_{0} \sim_{4}$ $q_{0}+\tau-t$ witnessed by $B\left(q_{0}, 16 \varrho\right)$. So the induced oscillation of $\Delta_{\tau-t} f(x)$ is less than 4 on $B\left(q_{0}, 16 \varrho\right)$. This implies that for every $y \in B\left(q_{0}, 8 \varrho\right)$ we have

$$
\left|\Delta_{y-q_{0}} \Delta_{\tau-t} f(x)\right|<8 \quad \mathcal{M} \text {-a.e. in } B\left(q_{0}, 8 \varrho\right) .
$$

Since $\Delta_{y-q_{0}} \Delta_{\tau-t} f(x)=\Delta_{\tau-t} \Delta_{y-q_{0}} f(x)$, we have

$$
\left|\Delta_{\tau-t} \Delta_{y-q_{0}} f(x)\right|<8 \quad \mathcal{M} \text {-a.e. in } B\left(q_{0}, 8 \varrho\right)
$$

for every $t, \tau \in E$ with $|t-\tau|<8 \varrho$. Since $\Delta_{y-q_{0}} f$ has the Baire property, $E-E$ is dense in $B(0,8 \varrho)$ and (11) shows that

$$
\left|\Delta_{z} \Delta_{y-q_{0}} f(x)\right|<8 \quad \mathcal{M} \text {-a.e. in } B\left(q_{0}, 4 \varrho\right)
$$

for every $z \in(E-E) \cap B(0,8 \varrho)$, we can apply Lemma 4.8 for $Z=(E-E)$ $\cap B(0,8 \varrho), g=\Delta_{y-q_{0}} f(x), \delta=4 \varrho$ and $K=8$. We conclude that the induced oscillation of $\Delta_{y-q_{0}} f(x)$ on $B\left(q_{0}, 4 \varrho\right)$ is less than 8 , that is, for every $y \in B\left(q_{0}, 4 \varrho\right)$ we have $q_{0} \sim_{8} y$ witnessed by $B\left(q_{0}, 4 \varrho\right)$.

Thus by Lemma 4.4,

$$
\forall x, y \in B\left(q_{0}, 4 \varrho\right) \quad x \sim_{16} y \text { witnessed by } B(x, 4 \varrho) .
$$

Let $f_{1}$ be the periodic extension of $\left.f\right|_{\left[q_{0}-\varrho / 2, q_{0}+\varrho / 2\right)}$, that is, for $x \in$ $\left[q_{0}-\varrho / 2, q_{0}+\varrho / 2\right)$ and $l \in \mathbb{Z}$ let

$$
f_{1}(x+l \varrho)=f(x) .
$$

We now show that for every $h \in \mathbb{R}$ the induced oscillation of $\Delta_{h} f_{1}$ is essentially bounded by a nonnegative constant $K$.

Let

$$
W=\{z \varrho: z \in \mathbb{Z}\},
$$

and let $h \in \mathbb{R}$. Since $\Delta_{h} f_{1}(t)$ is also periodic with period $\varrho$, it is enough to verify its boundedness for $t \in\left[q_{0}-\varrho / 2, q_{0}+\varrho / 2\right)$.

For $h \in W$ we have $\Delta_{h} f_{1}=0$. For $h \notin W$ let

$$
\widetilde{h}=h-\varrho[h / \varrho]
$$


where $[\cdot]$ stands for the integer part. For $q_{0}-\varrho / 2 \leq t<q_{0}+\varrho / 2-\widetilde{h}$ we have

$$
\Delta_{h} f_{1}(t)=\Delta_{\widetilde{h}} f(t) .
$$

By (12) for $x=t+\widetilde{h}$ and $y=t$ the induced oscillation of $\Delta_{\widetilde{h}} f(t)$ in $\left[q_{0}-\varrho / 2, q_{0}+\varrho / 2\right)$ is less than 16 . For $q_{0}+\varrho / 2-\widetilde{h} \leq t<q_{0}+\varrho / 2$ we have

$$
\Delta_{h} f_{1}(t)=\Delta_{\widetilde{h}-\varrho} f(t),
$$

and by (12) for $x=t+\widetilde{h}-\varrho$ and $y=t$ the induced oscillation of $\Delta_{\widetilde{h}-\varrho} f(t)$ in $\left[q_{0}-\varrho / 2, q_{0}+\varrho / 2\right)$ is also less than 16 .

So if we show that $\Delta_{\widetilde{h}} f(t)-\Delta_{\widetilde{h}-\varrho} f(t)$ is essentially bounded for $t \in$ $\left[q_{0}-\varrho / 2, q_{0}+\varrho / 2\right)$ by a nonnegative constant $R$ not depending on $h$, then it will follow that the induced oscillation of $\Delta_{h} f_{1}$ on $\left[q_{0}-\varrho / 2, q_{0}+\varrho / 2\right)$ is less than $K=R+16+16$.

To see this, observe first that

$$
\Delta_{\widetilde{h}} f(t)-\Delta_{\widetilde{h}-\varrho} f(t)=\Delta_{\varrho} f(t+\widetilde{h}-\varrho),
$$

a difference function of $f$ with the fixed difference $\varrho$ not depending on $h$. By (12) for $x=t+\widetilde{h}$ and $y=t+\widetilde{h}-\varrho$ this difference function $\Delta_{\varrho} f$ has bounded oscillation on $B\left(q_{0}, \varrho / 2\right)$, so it is essentially bounded there by a nonnegative constant $R$ not depending on $h$. Thus by (13),

$$
\Delta_{\widetilde{h}} f(t)-\Delta_{\widetilde{h}-\varrho} f(t)
$$

is also essentially bounded on $B\left(q_{0}, \varrho / 2\right)$ by $R$. This implies that the induced oscillation of $\Delta_{h} f_{1}$ is essentially bounded by a nonnegative constant $K$.

It is an easy computation that $f_{1}-f$ and $\Delta_{h} f_{1}$ have the Baire property for every $h \in \mathbb{R}$ (see the beginning of the proof of Theorem 1.1).

Since the induced oscillation of $\Delta_{h} f_{1}$ is at most $K$, one can find a "mean value" $D(h)$ of $\Delta_{h} f_{1}$, that is, a real such that

$$
\mathcal{N}\left(\left[\left|\Delta_{h} f_{1}-D(h)\right|<K\right]\right)=\mathbb{R} .
$$

Thus for any $h \in \mathbb{R}$ the set

$$
\left[\left|\Delta_{h} f_{1}-D(h)\right|>K\right]
$$

is meager, so for any fixed $u, v \in \mathbb{R}$ the set

$$
\left[\left|\left(D(u+v)-\Delta_{u+v} f_{1}\right)-\left(D(u)-\Delta_{u} f_{1}(\mathrm{Id}+v)\right)-\left(D(v)-\Delta_{v} f_{1}\right)\right|>3 K\right]
$$

is also meager. On the other hand,

$$
\begin{aligned}
& D(u+v)-D(u)-D(v) \\
& \quad=\left(D(u+v)-\Delta_{u+v} f_{1}\right)-\left(D(u)-\Delta_{u} f_{1}(\operatorname{Id}+v)\right)-\left(D(v)-\Delta_{v} f_{1}\right)
\end{aligned}
$$

is constant for every fixed $u, v \in \mathbb{R}$, so

$$
|D(u+v)-D(u)-D(v)| \leq 3 K .
$$


According to the Stability Theorem of Hyers this implies

$$
D=d+H
$$

where $d$ is a bounded function and $H$ is additive. Let $l=f_{1}-H$. Since

$$
\Delta_{h} l=\Delta_{h} f_{1}-H(h)=\Delta_{h} f_{1}-D(h)+d(h),
$$

$\Delta_{h} l$ is uniformly essentially bounded and has the Baire property for every $h \in \mathbb{R}$, so by Theorem 1.1,

$$
l=k+\phi
$$

where $k$ has the Baire property and $\phi$ is null.

With $g=f-f_{1}+k$ we finally have $f=g+H+\phi$ where $g$ has the Baire property, $H$ is additive and $\phi$ is null.

Acknowledgments. I especially thank Tamás Keleti for his overall help in writing this paper.

\section{References}

[1] M. Balcerzak, E. Kotlicka and W. Wojdowski, Difference functions for functions with the Baire property, Aequationes Math. 57 (1999), 278-287.

[2] R. Filipów, On the difference property of the family of functions with the Baire property, Acta Math. Hungar., submitted.

[3] T. Keleti, Difference functions of periodic measurable functions, Fund. Math. 15, (1998), 15-32.

[4] C. Kuratowski, Topologie, PWN-Polish Sci. Publ., Warszawa, 1958.

[5] M. Laczkovich, Functions with measurable differences, Acta Math. Acad. Sci. Hungar. 35 (1980), 217-235.

[6] - The difference property, in: Paul Erdős and his Mathematics, Springer, to appear.

[7] J. C. Oxtoby, Measure and Category, Springer, 1980.

Department of Applied Analysis

Eötvös Loránd University

Pázmány Péter sétány 1/C

1117 Budapest, Hungary

E-mail: matrait@renyi.hu

Received 26 November 2001;

in revised form 3 October 2002 